\title{
Atmospheric Retrieval Sensitivity Analysis for an Earth-Twin in the Future LIFE Mission
}

\section{Other Conference Item}

Author(s):

Konrad, Björn; Alei, Eleonora (iD; Quanz, Sascha Patrick (D); LIFE Initiative

Publication date:

2020-09

Permanent link:

https://doi.org/10.3929/ethz-b-000466669

Rights / license:

Creative Commons Attribution 4.0 International

Originally published in:

EPSC Abstracts 14, https://doi.org/10.5194/epsc2020-650 


\section{Atmospheric Retrieval Sensitivity Analysis for an Earth-Twin in the Future LIFE Mission}

Björn S. Konrad ${ }^{1}$, Eleonora Alei ${ }^{1}$, Sascha P. Quanz ${ }^{1}$, and the LIFE inititiative*

${ }^{1}$ ETH Zurich, Institute for Particle Physics and Astrophysics, Wolfgang Pauli Strasse 27, CH-8093 Zurich, Switzerland (konradb@student.ethz.ch)

${ }^{*}$ A full list of authors appears at the end of the abstract

\section{Summary}

LIFE is a space-based mid-infrared (MIR) nulling interferometer proposed in Quanz et al. (2018). We use a Bayesian retrieval framework to analyze how the information on the atmospheric structure extractable from MIR emission spectra of terrestrial exoplanets change with the covered wavelength range, signal to noise ratio (SNR) and spectral resolution (R). In combination with the science goals for the LIFE mission, these simulations will provide us with first quantitative estimates of key technical requirements needed for such a telescope.

\section{Context}

A long-term objective of exoplanet research is to investigate the atmospheric properties of small and rocky exoplanets. A driving force behind this effort is the potential identification of habitable worlds. This data will improve our understanding of the diversity of extra-solar planetary bodies. Currently planned space- and ground-based telescopes are unlikely to be sensitive enough to characterize a large number of such atmospheres.

Future space missions (e.g. NASA's LUVOIR [a] and HabEx [b] concepts), capable of characterizing exoplanet atmospheres in reflected light (in the optical and near-infrared (NIR) wavelength range), have been proposed. Complementary to these efforts, the LIFE mission proposed in Quanz et al. (2018), which is discussed in more detail in another submission to this conference by Angerhausen et al., targets the mid-infrared (MIR) thermal emission of exoplanets using a nulling interferometer. This wavelength range is of particular interest since it allows us to probe for atmospheric molecules not accessible in the optical or NIR range such as the biosignatures $\mathrm{CH}_{4}$ and $\mathrm{N}_{2} \mathrm{O}$ as well as $\mathrm{CO}$. Furthermore, MIR emission observations, in contrast to optical and NIR observations, are capable of accurately probing the temperature structure and the radius of exoplanets (Quanz et al. 2019). Both are hardly constrained in reflected light observations.

\section{Methods}

Our approach is based on the retrieval of simulated mock observations of Earth-twin exoplanets around solar-type stars. We generate the emission spectrum of an Earth twin using the 1D radiative transfer tool petitRADTRANS (Mollière et al., 2019). The model takes the surface gravity, the pressure-temperature (PT) profile and the atmospheric gas composition as input. We parametrize 
the standard Earth's PT profile with a 4th order polynomial and consider the line Absorption from $\mathrm{H}_{2} \mathrm{O}, \mathrm{CO}_{2}, \mathrm{CH}_{4}, \mathrm{O}_{3}, \mathrm{~N}_{2} \mathrm{O}$ and $\mathrm{CO}$ as well as collision-induced absorption from $\mathrm{N}_{2}$ and $\mathrm{O}_{2}$.

Then, we use the LifeSIM (Ottiger et al. in prep.) tool to estimate the wavelength-dependent SNR expected for observations with a LIFE-like telescope, considering noise sources from stellar leakage, local zodiacal dust, and exozodiacal dust. We consider an Earth twin located at a distance of $10 \mathrm{pc}$ from the Sun around a G2 star with 3 exozodis. We generate mock observations for the following grid of wavelength ranges, Rs and SNRs:

- Range $=3-20 \mu \mathrm{m}, 6-17 \mu \mathrm{m}$

- $\mathrm{R}=20,35,50$

- $\mathrm{SNR}=5,10,15$

Our retrieval mechanism is built upon the Multinest algorithm (Feroz et al., 2009). Given the Earth twin input spectrum and treating the LifeSIM noise as uncertainty on the spectrum, we retrieve the PT profile, the mass, the radius, the surface pressure as well as the abundances of the different atmospheric species. Performing a retrieval study of the spectra taking into account all possible combinations of the specified ranges in wavelength, R and SNR, provides first estimates for the technical requirements we need to meet to characterize Earth-like atmospheres.

\section{Results}

A preliminary result, and a proof of concept from testing of our retrieval framework, is shown in Figures 1 and 2. The input for the retrieval is an Earth-twin spectrum (range $=3-25 \mu \mathrm{m}, \mathrm{R}=1000$, $\mathrm{SNR}=50$ ) seen at a distance of $10 \mathrm{pc}$ from the Sun. Please note that for the results given below, only the planet's photon noise was considered and not yet additional noise sources.

Figure 1 shows that the PT profile parameters, as well as the surface temperature, are accurately retrieved by our retrieval suite. Figure 2 gives the retrieved posteriors for the planet's radius, mass and atmospheric abundances. The histogram in the top-right corner indicates the retrieved absolute abundance of $\mathrm{CO}_{2}$. We give all other abundances relative to the $\mathrm{CO}_{2}$ abundance. Additionally, our retrieval framework constrains the planet's radius with high fidelity.

These first results demonstrate that our retrieval suite provides reliable results. In-depth analyses of the results for the grid mentioned above are currently ongoing and will be presented at the conference. In addition, a comparison with literature results from simulations of reflected light observations will be discussed. 


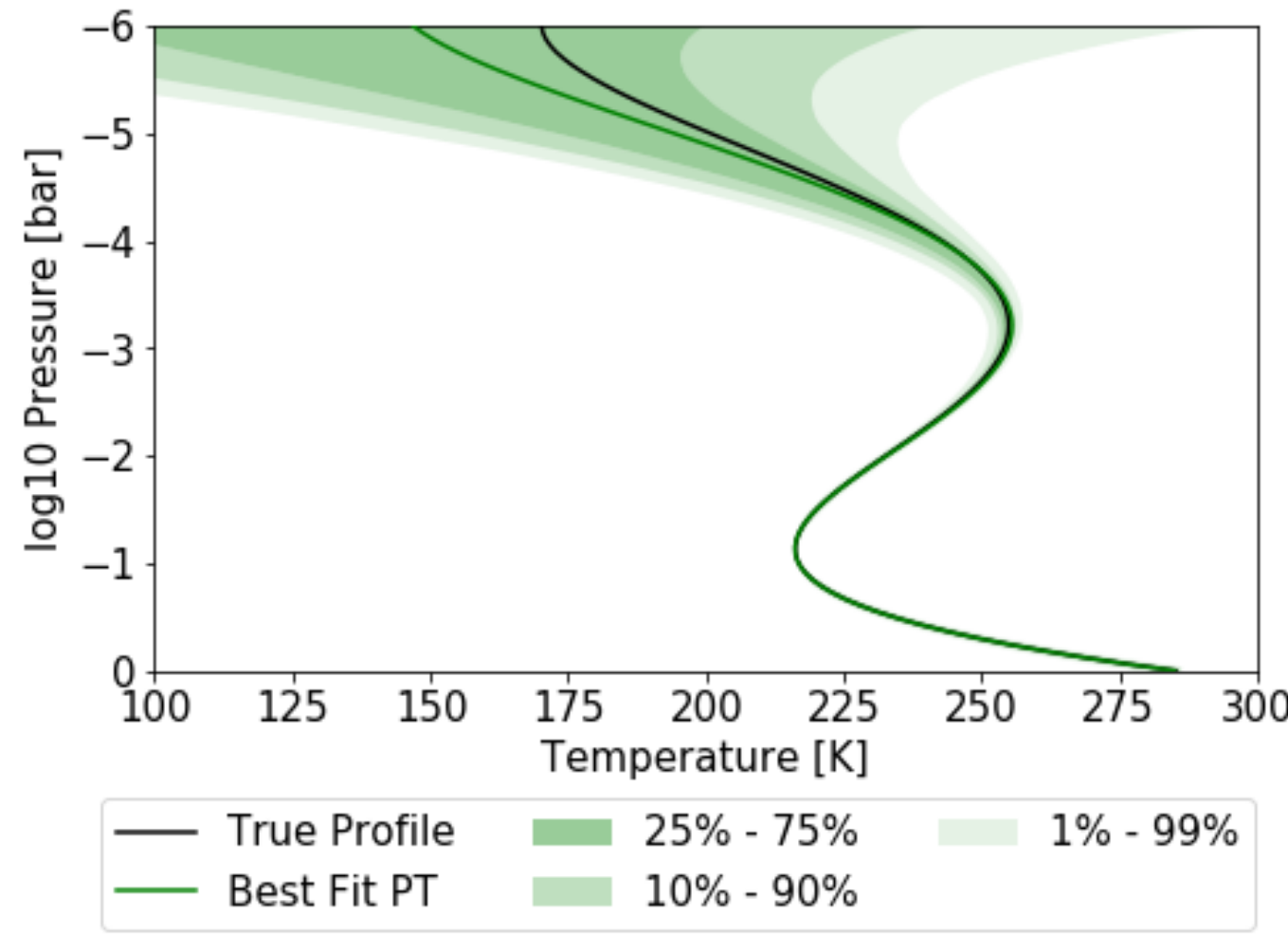

Figure 1: PT profile retrieved from an Earth-twin spectrum covering the wavelength range from 3 to $25 \mu \mathrm{m}$ with $\mathrm{R}=$ 1000 and SNR $=50$ at a distance of $10 \mathrm{pc}$. 


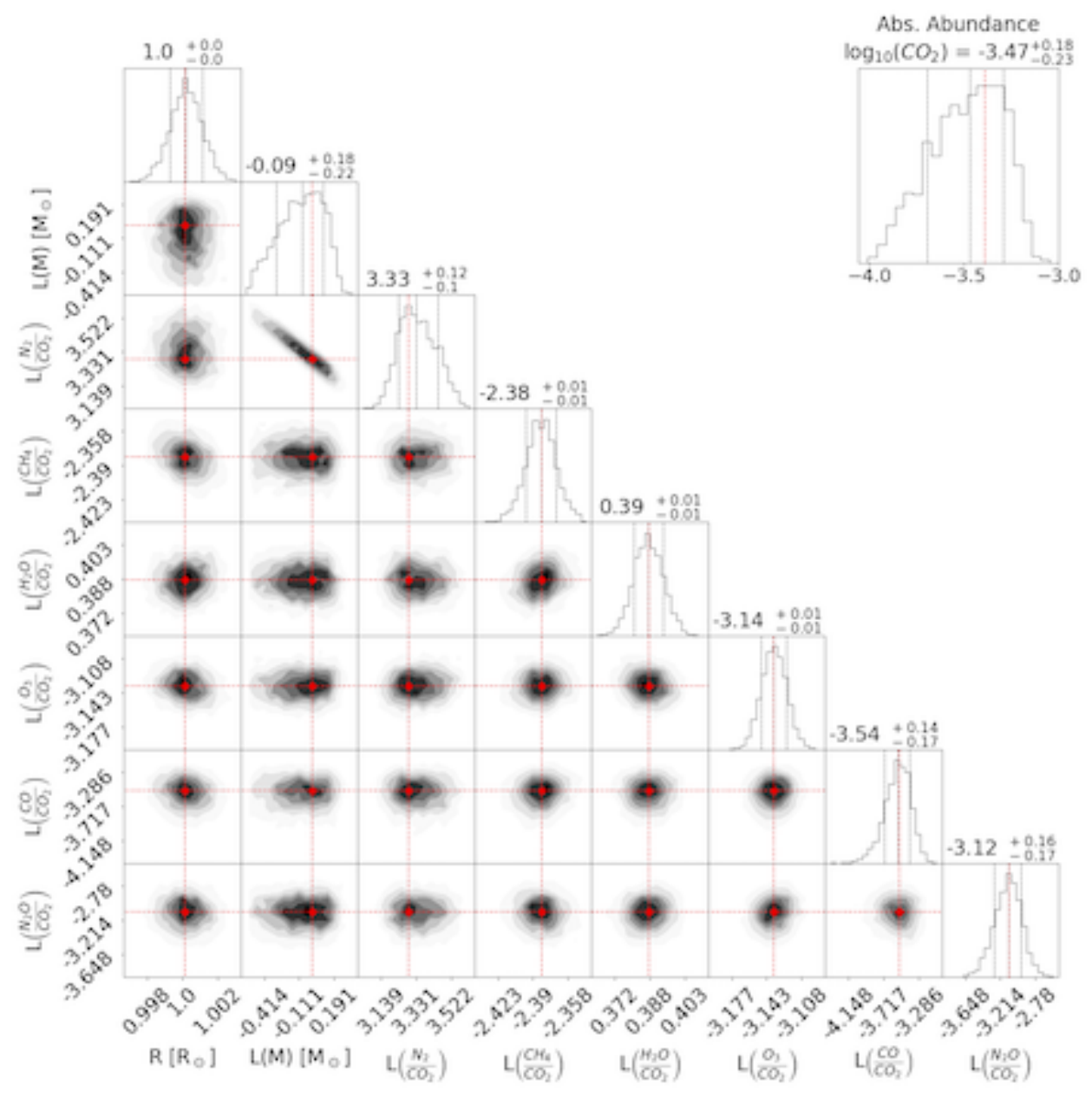

Figure 2: Radius, mass and abundances retrieved from an Earth-twin spectrum covering the wavelength range from 3 to $25 \mu \mathrm{m}$ with $R=1000$ and $S N R=50$ at a distance of $10 \mathrm{pc}$. $\mathrm{L}(\cdot)$ is an abbreviation for $\log _{10}(\cdot)$

\section{References}

Feroz, F., et al., 2009, MNRAS, 398(4):1601-1614

Mollière, P., et al., 2019, A\&A, 627:A67

Quanz, S. P., et al., 2018, Proc. SPIE, 107011I

Quanz, S. P. et al., 2019, arXiv e-prints arXiv:1908.01316

\section{Websites}

[a] https://www.luvoirtelescope.org

[b] https://www.jpl.nasa.gov/habex/

LIFE inititiative: www.life-space-mission.com 